\title{
O impacto do financiamento do PRONAF sobre indicadores agrícolas nas lavouras do Estado do Paraná: uma análise de dados em painel
}

\author{
Renato Alves Oliveira \\ Universidade de Ponta Grossa - Ponta Grossa - Paraná - Brasil \\ Luciano Ribeiro Bueno \\ Universidade de Ponta Grossa - Ponta Grossa - Paraná - Brasil
}

\section{Resumo}

O objetivo deste estudo foi analisar a influência do PRONAF - Programa Nacional de Fortalecimento da Agricultura Familiar sobre os indicadores agrícolas: produção, área plantada e produtividade das lavouras de subsistências beneficiadas pelo Programa no Estado do Paraná, no período de 2007 a 2012. A pesquisa utilizou um modelo de regressão de dados em painel. As lavouras de subsistência selecionadas que receberam benefícios do Programa no Estado foram: batata, arroz, cana-de-açúcar, feijão, mandioca, soja, café, trigo e milho. As análises da relação entre o montante de financiamento do PRONAF e os indicadores agrícolas apontaram que para a maioria das lavouras o PRONAF teve um efeito negativo, embora muito pequeno, sobre a produção, área plantada e produtividade. Contudo a influência do PRONAF no desempenho da produção e da produtividade do milho e da mandioca foi positivamente significativa.

Palavras-Chave: PRONAF. Paraná. Dados em painel. Indicadores agrícolas.

The impact of PRONAF financing on indicators in agricultural field of Paraná State: a panel data analysis

\begin{abstract}
The objective of this study was to analyze influence the PRONAF - Programa Nacional de Fortalecimento da Agricultura Familiar on the agricultural pointers: production, planted area and productivity of subsistences the farmings benefited for the Program in the State of the Paraná, the period 2007 to 2012. The research used a model the regression of panel in data. The selected subsistence of crops receiving benefited by the Program in State were: potatoes, rice, cane sugar, beans, cassava, soybeans, coffee, wheat and corn. The analysis of the relation enters the sum of financing of the PRONAF and the agricultural pointers pointed that for the majority of the farmings the Program had a negative effect, even so very small, on the production, planted area and productivity. However the influence of PRONAF on production and productivity performance of maize and cassava was significantly positively.
\end{abstract}


Keywords: PRONAF. State of the Paraná. Panel in data. Agricultural pointers.

\section{El impacto del financiamiento del PRONAF sobre indicadores agrícolas en los cultivos del Estado del Paraná: un análisis de datos de panel}

\section{Resumen}

El objetivo de este estudio fue analizar la influencia del PRONAF - Programa Nacional de Fortalecimiento de la Agricultura Familiar sobre los indicadores agrícolas: producción, área cultivada y productividad de los cultivos de subsistencia beneficiados por el Programa en el Estado de Paraná, en el período de 2007 a 2012. La investigación utilizó un modelo de regresión de datos de panel. Los cultivos de subsistencia seleccionados que recibieron beneficios del Programa en el Estado fueron: patata, arroz, caña de azúcar, frijoles, mandioca, soja, café, trigo y maíz. Los análisis de la relación entre el importe financiado por el PRONAF y los indicadores agrícolas señalaron que para la mayoría de los cultivos el PRONAF tuvo un efecto negativo, aunque muy pequeño, sobre la producción, área cultivada y productividad. Sin embargo, la influencia del PRONAF en el desempeño de la producción, la productividad del maíz y de la mandioca fue positivamente significativa.

Palabras-Clave: PRONAF. Paraná. Datos de panel. Indicadores agrícolas. Productividad agrícola.

\section{Introdução}

O PRONAF - Programa Nacional de Fortalecimento da Agricultura Familiar foi criado em 1995 como um Programa que busca promover o desenvolvimento sustentável do segmento rural constituído pelos agricultores familiares.

As grandes linhas de atuação do PRONAF, segundo Mattei (2006), são: i) financiamento da produção: através da liberação de recursos para custeio e investimentos em atividades produtivas em praticamente todos os municípios do Brasil; ii) financiamento de infra-estrutura e serviços municipais: através de apoio financeiro aos municípios para a execução de obras de infra-estrutura; iii) capacitação e profissionalização dos agricultores familiares; iv) financiamento da pesquisa e extensão rural: através da liberação de recursos para a geração e transferência de tecnologias aos agricultores familiares.

O Programa objetiva estimular a renda líquida e a qualidade de vida dos agricultores familiares através do aumento da produção agrícola e a geração de ocupações produtivas. Para tanto, propicia ao produtor um sistema de crédito rural de acesso simplificado (MDA, 2014).

Segundo Magalhães et al. (2006), o principal elemento de superação do PRONAF, em relação aos demais Programas de crédito, foi a ênfase no crescimento da capacidade produtiva a partir do suporte à capacitação tecnológica, assistência técnica e infra-estrutura. Além disso, ao financiar a produção de lavouras de subsistência o PRONAF adquire uma conotação democrática com distribuição de recursos entre os pequenos agricultores.

No Estado do Paraná as principais lavouras financiadas pelo PRONAF na modalidade custeio agrícola durante o período 2007 - 2012 foram, conforme o Anuário Estatístico de Crédito Rural (BACEN, 2015), soja e milho. Em menor proporção podem ser citadas as lavouras de café, mandioca, feijão e trigo. As lavouras menos expressivas são cana de açúcar, arroz e batata. 
São inúmeros os trabalhos realizados para avaliar os impactos do PRONAF na pobreza rural ou condições de vida dos agricultores familiares, como: estudos técnicos, científicos, com dados primários, secundários, desenvolvidos pelo governo ou comunidade acadêmica. Mas não existe um consenso sobre o real impacto deste Programa. No entanto, serão destacados nesta seção alguns posicionamentos relativos aos indicadores relevantes para o estudo aqui apresentado.

Há uma lacuna de análises que relacionem o impacto do financiamento do PRONAF aos indicadores agrícolas do Paraná, então esse estudo tem como objetivo analisar a influência do PRONAF sobre os indicadores: produção, área plantada e produtividade das lavouras de subsistências beneficiadas pelo Programa no Estado do Paraná.

Parte-se do pressuposto que esse financiamento tenha contribuído para a melhoria destes indicadores revelando o PRONAF como um programa de apoio ao desenvolvimento rural do Brasil.

Além dessa introdução, o artigo possui outras quatro seções. Na seção 2, apresenta-se uma breve revisão da literatura sobre a importância do PRONAF. Em seguida, na seção 3, apresentam-se pesquisas realizadas sobre a avaliação do PRONAF. Na seção 4, discute-se a metodologia utilizada para dados em painel. $\mathrm{Na}$ seção 5 , realiza-se a análise dos resultados. Por fim, na seção 6 , considerações finais do trabalho.

\section{Programa Nacional de Fortalecimento da Agricultura Familiar}

As unidades familiares necessitam de recursos financeiros para serem utilizados no custeio de atividades produtivas, em projetos de investimentos, em atividades de comercialização da produção e no suprimento das necessidades familiares dos agricultores, de modo a obter uma estratégia de desenvolvimento (SOUZA FILHO; BATALHA, 2005).

As políticas públicas por muitos anos acentuaram as diferenças no meio rural e agravaram os problemas econômicos e sociais enfrentados pela agricultura familiar por não levar em consideração a diversidade de situações verificadas no campo e pequenas cidades, as quais, em termos sociais, econômicos e políticos, diferem bastante.

De um modo geral, no início dos anos 1990, ainda não existia uma política pública nacional voltada para o segmento dos agricultores familiares, a qual se caracterizava de modo meramente instrumental e bastante impreciso no âmbito da burocracia estatal (CAZELLA;MATTEI; SCHNEIDER; 2004).

Segundo Mattei (2006), mesmo com os avanços da Constituição de 1988 no que tange à descentralização das ações estatais e criação de conselhos gestores que tinham como objetivo democratizar o acesso aos recursos públicos, no início dos anos 1990, ainda existia uma carência de políticas públicas voltadas às necessidades dos agricultores familiares. Alguns fatores foram decisivos para modificar o rumo das políticas de desenvolvimento rural na década de 90.

De acordo com Delgado (2001), a intervenção do Estado na agricultura tem uma longa tradição, tanto nos países desenvolvidos como nos subdesenvolvidos. $O$ objetivo da intervenção do Estado foi regular os mercados, garantir preços e rendas 
para os agricultores e estimular a produção doméstica com intuito de evitar escassez de produtos e a elevação dos preços internos.

Conforme Kageyama (1990) et al., é comum que três noções diferenciadas sejam utilizadas como sinônimas no processo de modernização da agricultura. Esses conceitos são: modernização da agricultura, industrialização da agricultura e formação dos complexos agroindustriais.

É importante lembrar que o Sistema Nacional de Crédito Rural foi instituído em 1965 pela Lei 4.829, de 05/11/65, e regulamentado pelo Decreto 58.380, de 10/05/66. Este era constituído pelo Banco Central, Banco do Brasil, Bancos Regionais de Desenvolvimento, Bancos Estaduais, Bancos Privados, Caixas Econômicas, Sociedades de Crédito, Financiamento e Investimento, Cooperativas e Órgãos de Assistência Técnica e Extensão Rural (LEITE, 2001).

Segundo Belik (2001), os anos 1990 tiveram como pano de fundo a abertura da economia por meio da diminuição das barreiras à importação. Essas medidas, por sua vez, colocaram a agricultura brasileira diante de uma situação diferente do período de modernização.

Nesse novo cenário, a partir do começo dos anos 1990, começaram a emergir os novos instrumentos de captação de recursos para a agricultura, ou seja, parte das fontes de financiamento públicas foi sendo substituída pelas fontes privadas. As fontes de financiamento privadas eram pautadas pelas interações financeiras dos agentes privados (empresas de processamento, empresas de máquinas e insumos agropecuários, agricultores integrados, traders, securitários). Esses novos mecanismos, os recursos privados, eram canalizados com o intuito de garantir a agilidade na comercialização física e no financiamento das culturas (BELIK, 2001).

A pressão exercida por meio das organizações e lutas pelos segmentos rurais menos favorecidos foi de extrema importância para a criação do PRONAF, em 1996. Esses segmentos menos favorecidos nunca tiveram acesso aos recursos do Sistema Financeiro Nacional. O programa nasceu com a finalidade de prover crédito agrícola e apoio institucional aos pequenos produtores rurais que vinham sendo excluídos das políticas públicas existentes e encontravam dificuldades para se manter no campo (CAZELLA; MATTEI; SCHNEIDER; 2004).

O apoio à agricultura familiar tem que ser pensado no âmbito do desenvolvimento local no qual os aspectos econômicos, sociais, ecológicos e culturais devam ser igualmente levados em conta, na busca de soluções não excludentes (CARNEIRO, 1997).

A modalidade financiamento da produção que engloba os recursos para custeio e investimento tem como objetivo o apoio financeiro aos agricultores familiares, segundo seis categorias. Essa diferenciação por categorias decorreu de um estudo realizado pelo convênio FAO/Incra no ano de 1999. O estudo recomendou a segmentação dos agricultores familiares beneficiários do Programa em grupos diferentes, utilizando o critério de renda bruta familiar anual. Esse procedimento permitiu que as regras de financiamento se adequassem à realidade de cada segmento social. Os encargos financeiros e os rebates (descontos) têm como objetivo auxiliar as parcelas com faixas menores de renda e com maiores dificuldades para produzir (Cazella; Mattei e Schneider, 2004). 
O PRONAF sofreu diversas formas de avaliação por vários segmentos de pesquisadores e analistas. A seguir serão apresentados alguns estudos ligados à temática.

\section{Avaliações do PRONAF}

Segundo Mattei et al. (2007) foi observado dois momentos distintos sobre o montante de recursos do crédito rural destinado aos agricultores familiares. Verificou-se que o volume de recursos disponibilizados nas safras agrícolas de 1999 a 2002 ficou praticamente estável, situando-se na faixa de $R \$ 2$ bilhões. Notou-se também que a partir de 2003 houve um aumento progressivo, sendo que na safra de 2005 o montante de recursos disponível aos agricultores familiares foi mais que o triplo em relação à safra de 1999. Isso sugere que este segmento de produtores rurais efetivamente está sendo priorizado pela política agrícola e de desenvolvimento rural. O plano da safra de 2006 reforça essa argumentação, uma vez que neste ano estavam orçados cerca de R\$ 8 bilhões para a agricultura familiar.

Cerqueira e Rocha (2002), afirmam que o crédito é um elemento essencial capaz de possibilitar um nível mais elevado de renda e o aumento do patrimônio familiar, garantindo não apenas a sobrevivência, mas a melhoria da qualidade de vida para as famílias rurais. No caso do PRONAF, ressaltaram que uma das muitas críticas é a distribuição desigual do crédito.

Figueiredo, Silva e Santos (2006) apontaram a necessidade de redistribuição dos recursos do PRONAF, a fim de beneficiar os agricultores de renda mais baixa. De forma geral, foi constatado que os estados com maior representatividade de agricultores familiares, ou maior número de estabelecimentos, como é o caso dos estados da região Sul, captam maior volume de recursos. Isso ocorre apesar de os produtores familiares destes estados possuírem, em média, maiores receitas. Assim, verifica-se uma concentração dos recursos do PRONAF em estados com produtores mais capitalizados.

Estudo sobre a Distribuição dos Recursos Financeiros do PRONAF para as regiões Sul e Nordeste do Brasil verificou que a distribuição de recursos financeiros do PRONAF ocorre de forma desigual entre as regiões brasileiras. Há uma concentração de recursos nos grupos do PRONAF - Crédito em que a faixa de renda é mais elevada na região Sul. A região recebe quase $50 \%$ do total que o programa direciona para a modalidade PRONAF - Crédito (COSTA, SOUSA e MASSUDA, 2010).

Analisando o impacto do montante do PRONAF em relação ao PIB Agropecuário do Território Vale do Mucri entre 2000 e 2007, Silva (2010) estimou que a variação de $1 \%$ do montante de créditos do PRONAF em nível, acarretou variação percentual de 0,056\% no valor do PIB Agropecuário no território do próximo ano. 0 autor ainda salienta que apesar do impacto positivo, o valor do coeficiente para o PIB Agropecuário foi baixo.

A figura 1 apresenta os recursos liberados e número de contratos do PRONAF sobre o custeio agrícola e pecuário. Observa-se que houve um comportamento contrário entre contratos e montante adquirido, isto sugere que o valor médio do contrato aumentou drasticamente ao longo do tempo sendo de $\mathrm{R} \$ 487,05 \mathrm{mil}$ em 2007 chegando a $R \$ 1,22$ milhões em 2012. A variação de números de contratos e do montante financiado durante o período foi de $-23,32 \%$ e $76,72 \%$, respectivamente. 
Figura 1- Números de contratos e Recursos liberados do PRONAF à nível de custeio no Brasil entre 2007 e 2012.

\begin{tabular}{|l|l|l|c|}
\hline 900.000 & & \\
850.000 \\
800.000 \\
750.000 \\
700.000
\end{tabular}

Fonte: Elaborado pelo autor a partir do Bacen (2015).

A figura 2 mostra os recursos liberados e número de contratos do PRONAF sobre o investimento agrícola e pecuário. Observa-se que houve uma relação direta entre contratos e montante adquirido. No entanto valor médio do contrato teve um aumento mais expressivo ao longo do tempo do que do custeio sendo de $\mathrm{R} \$ 336,28$ mil em 2007 chegando a $\mathrm{R} \$ 770,24$ mil em 2012. A variação de números de contratos e do montante financiado durante o período foi de $-9,72 \%$ e $132,63 \%$, respectivamente.

Figura 2- Números de contratos e Recursos liberados do PRONAF à nível de investimento no Brasil entre 2007 e 2012.

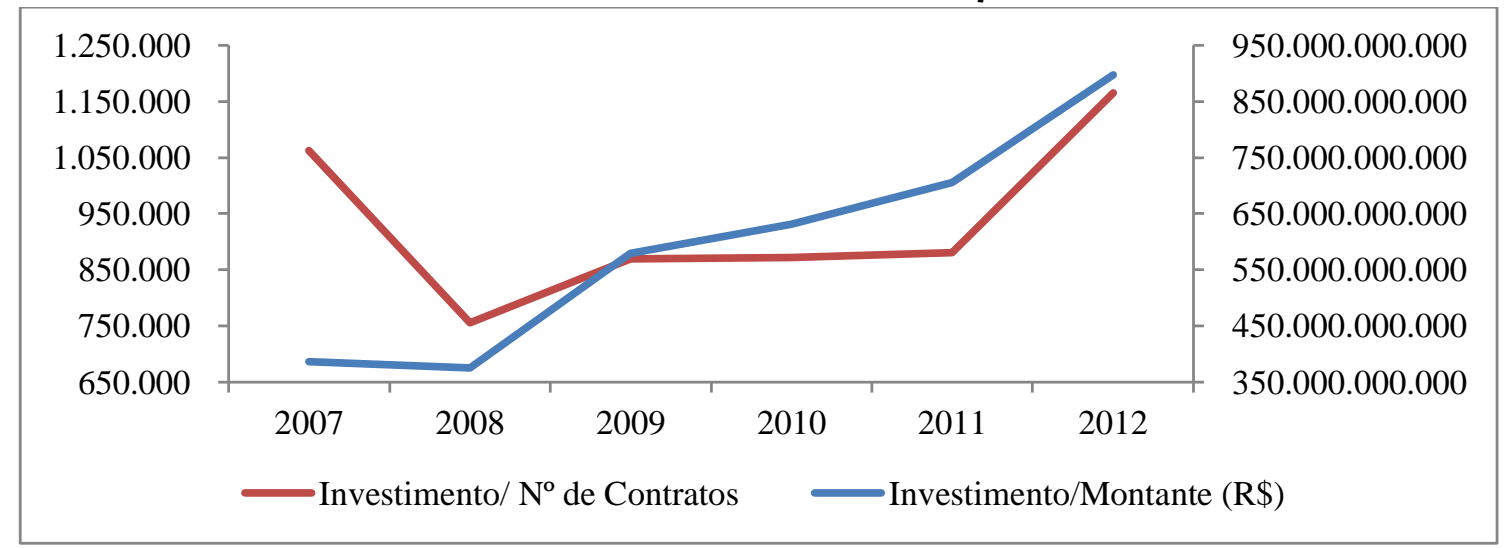

Fonte: Elaborado pelo autor a partir do Bacen (2015).

A partir dos dados do Bacen (2015) verifica-se que a região Sul obteve maior participação dos recursos liberados com 37,77\% em 2012. Segundo Felema, Raiher e Ferreira (2013) a região Sul detém uma participação elevada no valor agregado do setor, e uma das mais intensas produtividades do país.

Calzavara e Cruz (2013) observaram que o percentual de contratos do Sul era de $59,42 \%$, em 2001, e cai para 33,37\%, em 2011. Os agricultores sulistas continuam com maior representatividade, detendo, em 2011, 51,19\% dos valores disponibilizados. Essa representação é explicada, pois a região está, geograficamente, bem localizada e por deter agricultores familiares mais capitalizados.

\section{Metodologia}


A avaliação de uma política pública envolve uma série de obstáculos operacionais que variam desde a seleção dos indicadores analisados, obtenção dos dados até à escolha de um modelo estatístico adequado. No entanto, "é cada vez mais crescente o interesse neste tipo de análise com vistas a orientar a formulação, a execução, o acompanhamento e a avaliação de programas ou de projetos governamentais" (BRASIL, 2001, p.11). Nesta seção são descritos os procedimentos adotados na análise da influência do PRONAF nas principais lavouras de subsistência do Paraná.

\subsection{Descrição das variáveis e fonte dos dados}

A atividade rural no Brasil está distribuída entre a agricultura familiar e a empresarial, produzindo para o mercado interno e externo. No caso da agricultura familiar, tal segmento representa mais de $80 \%$ dos estabelecimentos no Paraná (IBGE, 2006).

Segundo o Anuário Estatístico do Crédito Rural realizado pelo Banco Central do Brasil as principais lavouras de subsistência praticadas pela agricultura familiar no Paraná são: arroz, batata, café, cana de açúcar, feijão, mandioca, milho, soja e trigo.

Em seguida foram coletados para cada lavoura os indicadores agrícolas de interesse:

- Produção: expressa em tonelada;

- Área plantada: expressa em ha;

- Produtividade: expressa em tonelada por ha.

Estes indicadores foram obtidos na Produção Agrícola Municipal (PAM) (2015) publicada pelo IBGE e foram escolhidos, pois são os mais susceptíveis ao financiamento da produção agrícola.

Os dados relativos ao PRONAF foram coletados junto à Secretaria de Agricultura Familiar (SAF) do Ministério do Desenvolvimento Agrário (dados agregados) (2014) e o Anuário Estatístico de Crédito Rural, disponibilizado pelo Banco Central (dados por lavoura). As variáveis consideradas foram:

- MontNegriante de investimentos realizados pelo PRONAF: expresso em R\$̦, corrigidos pelo IGP-DI, base dez / 2007, da Fundação Getúlio Vargas;

- Número de contratos.

Todas as séries utilizadas foram anuais. Devido à disponibilidade de dados foi estudado de 2007 a 2012 a evolução do montante e número de contratos do PRONAF de forma agregada, sem considerar as lavouras individualmente.

O financiamento da produção agrícola é de responsabilidade do PRONAF crédito, o qual concentra a maioria dos recursos liberados. De acordo com Sousa e Valente Júnior (2006) a linha de crédito do PRONAF, voltada para o financiamento da produção rural, configura-se em importante mecanismo de promoção do desenvolvimento sustentável do meio rural.

A pesquisa empregou o modelo de regressão de dados em painel para estudar o comportamento das variáveis, descrito a seguir.

\subsection{Método: Análise de dados em painel}


Um dos problemas mais comuns encontrados na estimação de modelos a partir de uma perspectiva de dados cross-section é o viés decorrente da omissão de variáveis relevantes na explicação do comportamento da variável dependente. Este problema foi claramente percebido na presente pesquisa quando se buscou mensurar a relação entre o montante de recursos do PRONAF e os indicadores agrícolas: produção, área e produtividade das lavouras de subsistência nos municípios do Paraná sem, no entanto, incluir nos modelos estimados as diferenças sistemáticas não observáveis entre os municípios tais como: características edáficas, vocação agrícola. Como estratégia para contornar o viés de omissão de variáveis optou-se por empregar a análise de dados em painel.

A análise de dados em painel é um método que requer um tipo específico de dados em que cada unidade de observação, indivíduo ou entidade, é observada em dois ou mais períodos de tempo. Um painel é dito balanceado quando as informações encontram-se disponíveis para todos os indivíduos em todos os períodos considerados na análise, e não balanceado quando não há disponibilidade de todas as observações para todos os indivíduos e períodos.

Segundo Berto (2007) as principais vantagens de se utilizar dados em painel são: redução de efeitos da colinearidade das variáveis independentes; mesclagem das informações de ordem dinâmica intertemporal com as informações individuais de cada entidade; controle da heterogeneidade individual; identificação e mensuração de efeitos não detectáveis através de cortes transversais e séries temporais isoladamente; construção e teste de modelos com comportamento mais complexo; e eliminação dos vieses resultantes da agregação de dados.

Como pode ser observado, existem interceptos diferentes para cada indivíduo e para cada período de tempo, o mesmo ocorrendo para os parâmetros associados às variáveis explicativas. Portanto, existem mais parâmetros a serem obtidos que o número de observações disponíveis, o que torna impossível a estimação do modelo. Para que o modelo se torne operacional são necessárias algumas suposições. Essas suposições são adotadas pelos três principais modelos de dados em painel (Wooldridge, 2007):

- Modelo de regressões aparentemente não relacionadas (SUR);

- Modelo de efeitos fixos e

- Modelo de efeitos aleatórios.

A seguir são descritos os modelos de efeitos fixos e efeitos aleatórios, os quais foram empregados nesta pesquisa.

\subsubsection{Modelo de efeitos fixos}

Segundo Hill, Griffiths e Judge (2003), o modelo de efeitos fixos busca controlar os efeitos das variáveis omitidas que variam de indivíduo para indivíduo porém, permanecem constantes ao longo do tempo. Neste modelo, o intercepto tem um valor diferente para cada indivíduo, mas é constante ao longo do tempo. Do lado dos coeficientes angulares, estes são constantes para todos os indivíduos e em todos os períodos analisados.

O modelo de efeitos fixos adota como suposições:

$$
\beta_{0 i t}=\beta_{0 i} \beta_{1 i t}=\beta_{1} \cdots \beta_{\text {kit }}=\beta_{k}
$$


Ao considerar que as variáveis omitidas permanecem constantes ao longo do tempo, o modelo incorpora um efeito fixo e passa a ser estimado, usando mínimos quadrados ordinários, através equação:

Em que:

$$
y_{i t}=C_{i}+\beta_{1} x_{1 i t}+\cdots+\beta_{k} x_{k i t}+e_{i t}(2)
$$

$\mathrm{C}_{\mathrm{i}}$ = interceptos a serem estimados para cada indivíduo. (Efeito fixo)

O efeito fixo na regressão capta todas as características não observadas dos indivíduos, as quais não variam ao longo do tempo. Pode ser interpretado como o efeito das variáveis omitidas no modelo.

A verificação das suposições do modelo é feita através de teste estatístico cujas hipóteses são:

$\mathrm{H}_{0}: \beta_{01}=\beta_{02}=\ldots=\beta_{0 k}$

$\mathrm{H}_{1}$ : os interceptos são diferentes

A estatística utilizada no teste é a F. Quando a hipótese nula é rejeitada, conclui-se que as suposições do modelo foram obedecidas.

\subsubsection{Modelo de efeitos aleatórios}

Hill, Griffiths e Judge (2003) afirmam que as suposições adotadas pelo modelo de efeitos aleatórios são as mesmas no modelo de efeitos fixos. A diferença entre os modelos consiste no tratamento dado aos interceptos. Enquanto no modelo de efeitos fixos os interceptos são parâmetros fixos, no modelo de efeitos aleatórios os interceptos são considerados variáveis al eatórias. No caso específico, desta pesquisa, o modelo considera que os municípios paranaenses são amostras aleatórias de uma população maior. Os interceptos passam a ser representados por:

$$
\beta_{0 i}=\overline{\beta_{0}}+C_{i}
$$

Ou seja, além do intercepto do modelo de efeitos fixos que capta as diferenças de comportamento entre os municípios existe ainda um intercepto, o qual é o componente populacional.

O modelo geral de efeitos aleatórios é dado por:

Sendo $\mu_{i t}=e_{i t}+C_{i}$

$$
y_{i t}=\overline{\beta_{0}}+\beta_{1} x_{1 i t}+\cdots+\beta_{k} x_{k i t}+\mu_{i t}(3)
$$

O novo termo de erro estocástico, $\mu_{\text {it }}$, apresenta quatro propriedades:

i) $\quad E\left(\mu_{i t}\right)=0$

ii) $\quad \operatorname{Var}\left(\mu_{\mathrm{it}}\right)=\sigma_{\mathrm{e}}^{2}+\sigma^{2} \mathrm{c}$

iii) $\operatorname{cov}\left(\mu_{\text {it }}, \mu_{\text {is }}\right)=\sigma^{2} c, \forall t \neq s$

iv) $\operatorname{cov}\left(\mu_{\mathrm{it}}, \mu_{\mathrm{jt}}\right)=0, \forall \mathrm{i} \neq \mathrm{j}$

A terceira propriedade aponta que os erros dos indivíduos ao longo do tempo são correlacionados (autocorrelação). Portanto o método dos mínimos quadrados não pode ser empregado na estimação da equação. Assim, no modelo de efeitos aleatórios, os melhores estimadores são obtidos pelo método de mínimos quadrados generalizados.

\subsubsection{Modelos adotados na pesquisa}

Nesta pesquisa foi utilizado um painel equilibrado para estimação dos modelos de efeitos fixos e aleatórios para cada um dos indicadores agrícolas 
referentes às lavouras de subsistência beneficiadas pelo PRONAF, as equações abaixo, representam os modelos de efeitos fixos:

$$
\begin{aligned}
& \log Y_{i t}=C_{i}+\beta_{1} \log X_{i t}+u_{i t} \\
& \log A_{i t}=C_{i}+\beta_{1} \log X_{i t}+u_{i t} \\
& \log R_{i t}=C_{i}+\beta_{1} \log X_{i t}+u_{i t}
\end{aligned}
$$

Sendo:

$Y_{i t}=$ produção do município $i$ no período $t$

$\mathrm{A}_{\mathrm{it}}=$ área plantada no município $\mathrm{i}$ no período $t$

$\mathrm{R}_{\mathrm{it}}=$ produtividade do município i no período $t$

$\mathrm{X}_{\mathrm{it}}=\mathrm{o}$ montante de recursos do PRONAF no município $\mathrm{i}$ no período $t$

$\beta_{1}=$ coeficiente associado ao montante de recursos no município i no período

$t$

$C_{i}=$ interceptos específicos para cada município i (efeito fixo)

$U_{i t}=$ erro aleatório do município i no período $t$

$\mathrm{i}=1, \ldots, \mathrm{n}$ e $\mathrm{t}=1, \ldots, \mathrm{T}$;

Os modelos de efeitos aleatórios foram representados por:

$\log Y_{i t}=\beta_{0}+\beta_{1} \log X_{i t}+v_{i t}$

$\log A_{i t}=\beta_{0}+\beta_{1} \log X_{i t}+v_{i t}$

$\log R_{i t}=\beta_{0}+\beta_{1} \log X_{i t}+v_{i t}$

Sendo:

$Y_{i t}=$ produção do município $i$ no período $t$

$A_{i t}=$ área plantada no município i no período $t$

$\mathrm{R}_{\mathrm{it}}=$ produtividade do município $i$ no período $t$

$X_{i t}=0$ montante de recursos do PRONAF no município i no período $t$

$\beta_{1}=$ coeficiente associado ao montante de recursos no município i no período

$t$

$\beta_{0}=$ o componente populacional para cada município i (efeito aleatório)

$v_{i t}=$ erro aleatório do município $i$ no período $t$

$i=1, \ldots$, n e $t=1, \ldots, T$;

Para cada lavoura o painel foi composto por um número de municípios diferentes dentro de um horizonte de tempo entre 2007 - 2012. A variável recursos do PRONAF com valor zero, foi excluída da amostra. As lavouras beneficiadas pelo PRONAF foram analisadas separadamente de modo que foram estimados 27 modelos de efeitos fixos e 27 modelos de efeitos aleatórios.

$\mathrm{Em}$ todas as regressões a heterocedasticidade foi tratada pelo método de correção no estimador da variância e co-variância de White. O software empregado para estimar os modelos foi o Eviews versão 8.0. A seguir a análise dos resultados.

\section{Resultados e Discussões}

Nesta seção apresentam-se os modelos econométricos propostos para mensurar a relação existente entre o montante de investimentos do PRONAF e a produção, área plantada e rendimento das lavouras de subsistência no Paraná, no período compreendido entre os anos 2007 - 2012. Antes de iniciar a análise, alguns pontos devem ser esclarecidos.

A estimação foi realizada a partir de um painel balanceado de dados, sendo considerados os modelos de efeitos fixos e efeitos aleatórios. Em todas as regressões o tratamento da heterocedasticidade e autocorrelação dos resíduos foi devidamente 
realizado. As tabelas com os resultados obtidos expõem o valor do coeficiente estimado, o teste de significância estatística deste coeficiente para o efeito fixo e o aleatório e a estatística de Hausman. O valor do intercepto ou constante foi omitido, pois não apresenta informações relevantes para a análise como explicado em Stock e Watson (2004).

Segundo Daher (2004), uma questão relevante no uso de modelos de dados em painel é a escolha de qual especificação: modelo de efeitos fixos ou modelo de efeitos aleatórios, explica melhor a relação estudada. Segundo Marques (2000) a escolha de uma ou outra especificação, depende do comportamento dos dados, no entanto, a literatura sugere a utilização do teste de especificação de Hausman. $O$ teste de Hausman verifica a existência de correlação entre os efeitos individuais das unidades de observação e as variáveis explicativas. Quando essa correlação não existe, o modelo de efeitos aleatórios é consistente e eficiente e o modelo de efeitos fixos pode ser consistente, porém ineficiente (Negri e Laplane, 2003). Assim, as hipóteses nula e alternativa do teste proposto são:

$\mathrm{H}_{0}$ : Não existe correlação entre os efeitos individuais e as variáveis explicativas (modelo de efeitos aleatório é mais adequado)

$\mathrm{H}_{\mathrm{A}}$ : Existe correlação entre os efeitos individuais e as variáveis explicativas (modelo de efeitos fixo é mais adequado)

A estimação do impacto do recurso do PRONAF sobre as variáveis área plantada, produção e produtividade da mandioca encontra-se na tabela 1. Para todas as variáveis ao comparar o modelo aleatório contra o efeito fixo, o teste de Hausman foi não significativo, então o efeito aleatório é o mais adequado. O PRONAF influenciou apenas significantemente a produção e a produtividade, com coeficiente positivo para todas as variáveis da mandioca nos municípios do Paraná. Desse modo podem-se interpretar as estimações da seguinte forma: mantidas as outras variáveis constantes, o aumento de $10 \%$ no montante do financiamento do PRONAF pode promover um aumento $0,52 \%$ na produção e $0,22 \%$ na produtividade de mandioca. $O$ impacto do PRONAF é pequeno sobre o aumento da produtividade e da produção de mandioca.

Tabela 1 -Estimação do impacto do PRONAF nos indicadores agrícolas da mandioca nos municípios do Paraná 2007-2012.

\begin{tabular}{cccc}
\hline Variável & $\begin{array}{c}\text { Coeficiente Efeito Fixo } \\
\text { (t-estatística) }\end{array}$ & $\begin{array}{c}\text { Coeficiente Efeito } \\
\text { Aleatório/(t-estatística) }\end{array}$ & $\begin{array}{c}\text { Hausman- } \chi^{2} / \\
(\mathrm{p} \text {-valor) }\end{array}$ \\
\hline Área Plantada & $0,010250(4.074562)$ & 0.023805 & 2,557655 \\
& & $(4,470288)$ & $(0,1098)$ \\
Produção & 0,040440 & $0,051960^{* *}$ & 1,743483 \\
& $(1,583924)$ & $(2,165540)$ & $(0,1867)$ \\
Produtividade & $0,030190^{*}(4,238755)$ & $0.022376^{*}$ & 2,351314 \\
& & $(4.496890)$ & $(0,1252)$ \\
\hline
\end{tabular}

Fonte: Estimado pelo autor * significativo a $1 \%, * *$ significativo a $5 \%$.

A estimação do impacto do recurso do PRONAF sobre as variáveis área, plantada, produção e produtividade do milho encontra-se na tabela 2. Ao comparar as variáveis sobre o modelo aleatório contra o efeito fixo, o teste de Hausman foi significativo para área plantada e produção, sendo o efeito fixo o mais adequado, enquanto é preferível o efeito aleatório para produtividade. O PRONAF influenciou apenas significantemente a produção e a produtividade, com coeficiente positivo 
para todas as variáveis do milho nos municípios do Paraná. Desse modo podem-se interpretar as estimações da seguinte forma: mantidas as outras variáveis constantes, o aumento de $10 \%$ no montante do financiamento do PRONAF pode promover um aumento $0,64 \%$ na produção e $0,76 \%$ na produtividade do milho. $O$ impacto do PRONAF sobre o aumento da produtividade e da produção do milho foi baixo.

Tabela 2-Estimação do impacto do PRONAF nos indicadores agrícolas do milho nos municípios do Paraná 2007-2012.

\begin{tabular}{|c|c|c|c|}
\hline Variável & $\begin{array}{l}\text { Coeficiente } \\
\text { Efeito Fixo (t- } \\
\text { estatística) }\end{array}$ & $\begin{array}{l}\text { Coeficiente } \\
\text { Efeito Aleatório/(t- } \\
\text { estatística) }\end{array}$ & $\begin{array}{l}\text { Hausman- } \\
\chi^{2} / \\
\text { (p-valor) }\end{array}$ \\
\hline Área Plantada & $\begin{array}{l}0.044146 \\
(1.194137)\end{array}$ & $\begin{array}{c}0.305096^{*} \\
(11.01822)\end{array}$ & $\begin{array}{l}113.504775^{*} \\
(0,0000)\end{array}$ \\
\hline Produção & $\begin{array}{c}0.064874^{* *} \\
(2.468733)\end{array}$ & $\begin{array}{c}0.229914 \\
(10.10357)\end{array}$ & $\begin{array}{l}157.690651^{*} \\
(0,0000)\end{array}$ \\
\hline Produtividade & $\begin{array}{c}0.020729 \\
(0.580360)\end{array}$ & $\begin{array}{l}0.076949 * \\
(5.765383)\end{array}$ & $\begin{array}{l}2.879740 \\
(0.0897)\end{array}$ \\
\hline
\end{tabular}

Fonte: Estimado pelo autor * significativo a $1 \%$, ** significativo a $5 \%$.

A estimação do impacto do recurso do PRONAF sobre as variáveis área plantada, produção e produtividade da soja encontra-se na tabela 3. Ao comparar as variáveis sobre o modelo aleatório contra o efeito fixo, o teste de Hausman foi significativo para todas as variáveis, sendo o efeito fixo o mais adequado. O PRONAF influenciou apenas significantemente e negativamente a produção e a produtividade nos municípios do Paraná. Desse modo podem-se interpretar as estimações da seguinte forma: mantidas as outras variáveis constantes, o aumento de $10 \%$ no montante do financiamento do PRONAF pode promover uma redução 1,06\% na produção e $1,11 \%$ na produtividade da soja.

Tabela 3-Estimação do impacto do PRONAF nos indicadores agrícolas da soja nos municípios do Paraná 2007-2012.

\begin{tabular}{cccc}
\hline Variável & $\begin{array}{c}\text { Coeficiente Efeito Fixo } \\
\text { (t-estatística) }\end{array}$ & $\begin{array}{c}\text { Coeficiente Efeito } \\
\text { Aleatório/(t-estatística) }\end{array}$ & $\begin{array}{c}\text { Hausman- } \chi^{2} / \\
(\mathrm{p} \text {-valor) }\end{array}$ \\
\hline Área Plantada & 0.004676 & $0.050098^{*}$ & $83.281941^{*}$ \\
& $(0.275111)$ & $(3.082738)$ & $(0.0000)$ \\
Produção & $-0.106336^{*}$ & -0.012640 & $112.455711^{*}$ \\
Produtividade & $(-4.569870)$ & $(-0.587214)$ & $(0.0000)$ \\
& $-0.111012^{*}$ & -0.002494 & $59.787405^{*}$ \\
& $(-7.515666)$ & $(-0.541575)$ & $(0.0000)$ \\
\hline
\end{tabular}

Fonte: Estimado pelo autor * significativo a $1 \%$

A estimação do impacto do recurso do PRONAF sobre as variáveis área plantada, produção e produtividade do trigo encontra-se na tabela 4. Ao comparar as variáveis sobre o modelo aleatório contra o efeito fixo, o teste de Hausman foi significativo para todas as variáveis, sendo o efeito fixo o mais adequado. O PRONAF influenciou significantemente e negativamente a área, a produção e a produtividade nos municípios do Paraná. Desse modo, podem-se interpretar as estimações da seguinte forma: mantidas as outras variáveis constantes, o aumento de $10 \%$ no montante do financiamento do PRONAF pode promover uma redução de $2,9 \%$ na área, $3,48 \%$ na produção e $0,48 \%$ na produtividade do trigo. 
Tabela 4-Estimação do impacto do PRONAF nos indicadores agrícolas do trigo nos municípios do Paraná 2007-2012.

\begin{tabular}{cccc}
\hline Variável & $\begin{array}{c}\text { Coeficiente Efeito Fixo } \\
(\mathrm{t}-\mathrm{estat} \text { stica) }\end{array}$ & $\begin{array}{c}\text { Coeficiente Efeito } \\
\text { Aleatório/(t-estatística) }\end{array}$ & $\begin{array}{c}\text { Hausman- } \chi^{2} / \\
(\mathrm{p} \text {-valor) }\end{array}$ \\
\hline Área Plantada & $-0.299785^{*}$ & $0.163092^{*}$ & $27.391268^{*}$ \\
& $(6.289088)$ & $(-4.090052)$ & $(0.0000)$ \\
Produção & $-0.348148^{*}$ & $-0.190919^{*}$ & $32.543312^{*}$ \\
& $(-6.937751)$ & $(-4.587004)$ & $(0.0000)$ \\
Produtividade & $-0.048363^{*}$ & -0.005274 & $6.784325^{*}$ \\
& $(-2.754798)$ & $(-0.897379)$ & $(0.0092)$ \\
\hline
\end{tabular}

Fonte: Estimado pelo autor * significativo a $1 \%$.

A estimação do impacto do recurso do PRONAF sobre as variáveis área plantada, produção e produtividade do arroz encontra-se na tabela 5. Ao comparar as variáveis sobre o modelo aleatório contra o efeito fixo, o teste de Hausman foi significativo para variável área plantada, nesse sentido o efeito fixo o mais adequado. O PRONAF influenciou significantemente e negativamente a área, a produção e a produtividade nos municípios do Paraná. Desse modo, podem-se interpretar as estimações da seguinte forma: mantidas as outras variáveis constantes, o aumento de $10 \%$ no montante do financiamento do PRONAF pode promover uma redução de $2,4 \%$ na área, $2,6 \%$ na produção e $0,8 \%$ na produtividade do arroz.

Tabela 5 - Estimação do impacto do PRONAF nos indicadores agrícolas do arroz nos municípios do Paraná 2007-2012.

\begin{tabular}{cccc}
\hline Variável & $\begin{array}{c}\text { Coeficiente Efeito Fixo } \\
\text { (t-estatística) }\end{array}$ & $\begin{array}{c}\text { Coeficiente Efeito } \\
\text { Aleatório/(t-estatística) }\end{array}$ & $\begin{array}{c}\text { Hausman- } \chi^{2} / \\
(\mathrm{p} \text {-valor) }\end{array}$ \\
\hline Área Plantada & $-0.246207^{*}$ & $-0.187396^{*}$ & 5.736206 \\
& $(-5.390984)$ & $(-4.866527)$ & $(0.0166)$ \\
Produção & $-0.320431^{*}$ & $-0.268761^{*}$ & 3.531298 \\
& $(-6.165691)$ & $(-6.094290)$ & $(0.0602)$ \\
Produtividade & $-0.074224^{*}$ & $-0.082246^{*}$ & 0.418904 \\
& $(-3.896178)$ & $(-5.684937)$ & $(0.5175)$ \\
\hline
\end{tabular}

Fonte: Estimado pelo autor * significativo a $1 \%$.

A estimação do impacto do recurso do PRONAF sobre as variáveis área plantada, produção e produtividade da batata encontra-se na tabela 6 . Ao comparar as variáveis sobre o modelo aleatório contra o efeito fixo, o teste de Hausman não foi significativo para nenhuma das variáveis analisadas, nesse sentido o modelo de efeito aleatório é o mais adequado. O PRONAF não influenciou significantemente nenhuma das variáveis analisadas.

Tabela 6 - Estimação do impacto do PRONAF nos indicadores agrícolas da batata nos municípios do Paraná 2007-2012.

\begin{tabular}{cccc}
\hline Variável & $\begin{array}{c}\text { Coeficiente Efeito Fixo } \\
\text { (t-estatística) }\end{array}$ & $\begin{array}{c}\text { Coeficiente Efeito } \\
\text { Aleatório/(t-estatística) }\end{array}$ & $\begin{array}{c}\text { Hausman- } \chi^{2} / \\
\text { ( } \mathrm{p} \text {-valor) }\end{array}$ \\
\hline Área Plantada & 0.021014 & 0.019864 & 0.122982 \\
& $(0.704777)$ & $(0.670275)$ & $(0.7258)$ \\
Produção & 0.017143 & 0.015691 & 0.198971 \\
& $(0.533489)$ & $(0.490824)$ & $(0.6556)$ \\
Produtividade & -0.003871 & -0.004794 & 0.717573 \\
\hline
\end{tabular}


$(-0.501159)$

$(-0.626863)$

$(0.3969)$

Fonte: Estimado pelo autor.

A estimação do impacto do recurso do PRONAF sobre as variáveis área plantada, produção e produtividade do café encontra-se na tabela 7. Ao comparar as variáveis sobre o modelo aleatório contra o efeito fixo, o teste de Hausman foi significativo para todas as variáveis, nesse sentido o efeito fixo é o mais adequado. $O$ PRONAF influenciou significantemente e negativamente a área, a produção e a produtividade nos municípios do Paraná. Desse modo, podem-se interpretar as estimações da seguinte forma: mantidas as outras variáveis constantes, o aumento de $10 \%$ no montante do financiamento do PRONAF pode promover uma redução de $0,3 \%$ na área, $0,3 \%$ na produçãoe praticamente nenhum efeito na produtividade do café.

Tabela 7 - Estimação do impacto do PRONAF nos indicadores agrícolas do café nos municípios do Paraná 2007-2012.

\begin{tabular}{cccc}
\hline Variável & $\begin{array}{c}\text { Coeficiente Efeito Fixo } \\
\text { (t-estatística) }\end{array}$ & $\begin{array}{c}\text { Coeficiente Efeito } \\
\text { Aleatório/(t-estatística) }\end{array}$ & $\begin{array}{c}\text { Hausman- } \chi^{2} / \\
(p-v a l o r)\end{array}$ \\
\hline Área Plantada & $-0.031154^{*}$ & $-0.030966^{*}$ & 9.296631 \\
& $(-13.30599)$ & $(-13.23043)$ & $(0.0023)$ \\
Produção & $-0.038282^{*}$ & $-0.037896^{*}$ & 13.932030 \\
& $(-12.24597)$ & $(-12.12912)$ & $(0.0002)$ \\
Produtividade & $-0.007089^{*}$ & $-0.005996 *$ & $(0.0000)$ \\
& $(-3.805829)$ & $(-3.241190)$ & \\
\hline
\end{tabular}

Fonte: Estimado pelo autor * significativo a $1 \%$.

A estimação do impacto do recurso do PRONAF sobre as variáveis área plantada, produção e produtividade dacana de açúcar encontra-se na tabela 8 . Ao comparar as variáveis sobre o modelo aleatório contra o efeito fixo, o teste de Hausman foi significativo para todas as variáveis, nesse sentido o efeito fixo é o mais adequado. O PRONAF influenciou significantemente e negativamente apenas a produtividade nos municípios do Paraná. Desse modo, podem-se interpretar as estimações da seguinte forma: mantidas as outras variáveis constantes, o aumento de $10 \%$ no montante do financiamento do PRONAF pode promover uma redução de $0,1 \%$ na produtividade.

Tabela 8 - Estimação do impacto do PRONAF nos indicadores agrícolas da cana nos municípios do Paraná 2007-2012.

\begin{tabular}{cccc}
\hline Variável & $\begin{array}{c}\text { Coeficiente Efeito Fixo } \\
\text { (t-estatística) }\end{array}$ & $\begin{array}{c}\text { Coeficiente Efeito } \\
\text { Aleatório/(t-estatística) }\end{array}$ & $\begin{array}{c}\text { Hausman- } \chi^{2} / \\
(\mathrm{p} \text {-valor) }\end{array}$ \\
\hline Área Plantada & 0.031708 & -0.064907 & 53.686758 \\
$(0.0000)$ & $(-1.851745)$ & 53.302114 \\
Produção & $(0.3973)$ & $-0.081451^{*}$ & $(0.0000)$ \\
Produtividade & $(0.010073$ & $(-2.283493)$ & 14.185174 \\
& $-0.0196427)$ & $-0.042212^{*}$ & $(0.0002)$ \\
\hline
\end{tabular}

Fonte: Estimado pelo autor * significativo a $1 \%$. 
A estimação do impacto do recurso do PRONAF sobre as variáveis área plantada, produção e produtividade do feijão encontra-se na tabela 9. Ao comparar as variáveis sobre o modelo aleatório contra o efeito fixo, o teste de Hausman foi significativo para todas as variáveis, nesse sentido o efeito fixo é o mais adequado. $O$ PRONAF influenciou significantemente e negativamentea área plantada e produção nos municípios do Paraná. Desse modo, podem-se interpretar as estimações da seguinte forma: mantidas as outras variáveis constantes, o aumento de $10 \%$ no montante do financiamento do PRONAF pode promover uma redução de 1,0\% na área plantada e também 1,0\% na produção.

Tabela 9 - Estimação do impacto do PRONAF nos indicadores agrícolas do feijão nos municípios do Paraná 2007-2012.

\begin{tabular}{cccc}
\hline Variável & $\begin{array}{c}\text { Coeficiente Efeito Fixo } \\
\text { (t-estatística) }\end{array}$ & $\begin{array}{c}\text { Coeficiente Efeito } \\
\text { Aleatório/(t-estatística) }\end{array}$ & $\begin{array}{c}\text { Hausman- } \chi^{2} / \\
(\mathrm{p} \text {-valor) }\end{array}$ \\
\hline Área Plantada & $-0.109236^{*}$ & 0.056861 & 101.822413 \\
& $(-3.018903)$ & $(1.764594)$ & $(0.0000)$ \\
Produção & $-0.102232^{*}$ & $0.080154^{*}$ & $(0.0000)$ \\
& $(-2.573151)$ & $(0.0245)$ & 32.776305 \\
Produtividade & 0.007003 & 0.071270 & $(0.0000)$ \\
& $(0.445615)$ & $(6.479616) *$ & \\
\hline
\end{tabular}

Fonte: Estimado pelo autor * significativo a $1 \%$.

No Paraná, o Programa gerou impacto negativo e significativo para a as lavouras beneficiadas: soja, trigo, arroz, café, cana de açúcar e feijão. Os recursos do PRONAF para batata sobre os indicadores não foram significativos. Apenas para a mandioca e o milho houve impacto positivo e significativo do financiamento. Em geral, foi observado que o impacto do PRONAF sobre as variáveis foi pequeno, talvez seja devido ao baixo nível da produção das lavouras analisadas em alguns municípios paranaenses.

\section{Considerações finais}

Este Programa tem como principal proposta promover o desenvolvimento sustentável do segmento rural constituído pelos agricultores familiares, com vistas a propiciar um sistema de crédito rural de acesso simplificado para a promoção e aumento da renda familiar, criação de novos postos de trabalho no campo e estímulo à produção de alimentos e assim, reduzir a pobreza do campo.

Analisados os indicadores agrícolas das principais lavouras de subsistência do Estado do Paraná houve um aumento geral no montante de recursos para a maioria das lavouras financiadas pelo PRONAF ao longo do período analisado. A lavoura de milho foi a que recebeu maior volume de financiamento concedido pelo PRONAF a partir de 2007.

A partir deste estudo pode-se verificar que indicadores como produção, área e produtividade sobre a maioria das lavouras analisadas têm sido negativamente e significantemente influenciados pelo Programa no Estado, ou seja, o PRONAF pode não está conseguindo aumentar a capacidade produtiva dos agricultores familiares.

Com exceção da mandioca e do milho ambas apresentaram impacto significativo e positivo do financiamento do PRONAF sobre os indicadores produção 
e produtividade. Isso sugere que as perspectivas desse Programa para a redução da pobreza rural estão de fato ocorrendo.

É importante ressaltar que apesar de não serem significativas, algumas estimações apresentaram impactos positivos dos recursos do PRONAF sobre os indicadores tanto para efeito fixo quanto para o aleatório.

Portanto, estes resultados apresentam certas limitações referentes aos dados disponíveis da pesquisa, pois, por exemplo, há dúvidas se o montante de recursos utilizado está realmente sendo empregado nas lavouras de subsistência. As condições climáticas do Estado como precipitações irregulares, podem ter inviabilizado o financiamento da produção.

Recomendam-se estudos de casos para verificar se há uma relação positiva do financiamento do PRONAF sobre estes e outros indicadores não analisados.

\section{REFERÊNCIAS}

BACEN- Banco Central do Brasil. Anuário Estatístico de Crédito Rural. Disponível em: http://www.mda.gov.br/index. Acesso em: 15/02/2015.

BRASIL. TRIBUNAL DE CONTAS DA UNIÃO. Técnicas de Auditoria: Marco Lógico / Tribunal de Contas da União. - Brasília: TCU, Secretaria de Fiscalização e Avaliação de Programas de Governo, 2001. Disponível em:

http://portal.tcu.gov.br/biblioteca-digital/tecnica-de-auditoria-marco-logico.htm Acesso em: 26 ago. 2015.

BELIK,Walter. PRONAF: avaliação da operacionalização do programa. Campinas. Universidade estadual de Campinas. NEA, 2001.

BERTO, Maria Eduarda Gouvêa. Determinantes da estrutura de capital de empresas brasileiras: uma análise de dados em painel (2004-2006) discriminando entre as maiores e menores empresas da BOVESPA. Dissertação (Mestrado em Administração)-Pontifícia Universidade Católica do Rio de Janeiro, Rio de Janeiro, 2007. $114 \mathrm{f}$.

CALZAVARA, G.; CRUZ,A.C. Análise da concentração de recursos do PRONAF. Sociedade e Desenvolvimento Rural on line, v.7, n. 4, 2013.

CARNEIRO, Maria José. Política pública e agricultura familiar: uma leitura do PRONAF. Estudos Sociedade e Agricultura. 1997.

CAZELLA, A. A.; MATTEI, L.; SCHNEIDER, S.. Histórico, caracterização e dinâmica recente do PRONAF: Programa Nacional de Fortalecimento da Agricultura Familiar, In: Congresso da Sociedade Brasileira de Economia e Sociologia Rural, Cuiabá, jul. 2004.

CERQUEIRA, P.S. e ROCHA, A.G. A agricultura familiar e o PRONAF: elementos para uma discussão. Bahia Análise \& Dados, Salvador, v. 12, n. 3, p. 105-117, 2002. 
COSTA, C. K. F.; SOUSA, M. M.M. ; MASSUDA, E. M. . Distribuição dos recursos financeiros do PRONAF para as regiões sul e nordeste do Brasil: uma análise sob teoria de restrição de crédito. Rama: Revista em Agronegócio e Meio Ambiente, v. 3, p. 213-232, 2010.

DAHER, C. E.. Testes Empíricos de Teorias Alternativas sobre a Determinação da Estrutura de Capital das Empresas Brasileiras. Dissertação apresentada ao Programa Multiinstitucional e Inter-Regional de Pós-Graduação em Ciências Contábeis UnB, Brasília, 2004.

DELGADO, Nelson Giordano. Política econômica, ajuste externo e agricultura. In: LEITE, Sérgio. (org.). Políticas Públicas e Agricultura no Brasil. Porto Alegre. Editora da Universidade, UFRGS, 2001.

FELEMA, J.;RAIHER,A.P. e FERREIRA, C.R. Agropecuária Brasileira: desempenho regional e determinantes de produtividade. RESR, Piracicaba-SP, Vol. 51, Nº 3, p. 555574, Jul/Set 2013.

FIGUEIREDO, A. M., SILVA, M. A. P. e SANTOS, M.L. Distribuição Estadual dos Recursos do PRONAF e sua Comparação com a Renda dos Produtores Familiares. Anais do XLIV Congresso SOBER, Julho de 2006.

HILL, R.C.;GRIFFITHS, W.E.; JUDGE,G.G. Econometria. $2^{\text {a }}$ ed. São Paulo: Editora Saraiva. 2003.

IBGE- Instituto Brasileiro de Geografia e Estatística. CENSO Agropecuário. 2006. KAGEYAMA, Angela. et al. O novo padrão agrícola brasileiro: do complexo rural aos complexos agroindustriais. In: Guilherme da Costa Deldago et al., Agricultura e Política Públicas. Brasília, IPEA, 1990.

LEITE, Sergio. (org.). Políticas Públicas e Agricultura no Brasil. Porto Alegre. Editora da Universidade, UFRGS, 2001.

MAGALHÃES, A. M., et al. A experiência recente do PRONAF em Pernambuco: uma análise por meio de propensity score. Economia Aplicada, Ribeirão Preto, vol.10 n.1, 2006.

MARQUES, L. D. Modelos dinâmicos com dados em painel: revisão de literatura. Porto: Faculdade de Economia do Porto, 2000.

MATTEI, Lauro. PRONAF 10 anos: mapa da produção acadêmica. Brasília: MDA, 2006. .Uma análise dos impactos do PRONAF sobre as economias locais nas regiões Nordeste, Sudeste e Norte do Brasil. XLV CONGRESSO DA SOBER. Sociedade Brasileira de Economia, Administração e Sociologia Rural. Londrina, 2007. 
MINISTÉRIO DO DESENVOLVIMENTO AGRÁRIO. Como o agricultor familiar pode conseguir e manter o financiamento rural e como se dá à relação com os bancos. Disponível em: http://www.mda.gov.br/index. Acesso em 22 de fevereiro 2014.

NEGRI, F. de; LAPLANE, M. F. Impactos das empresas estrangeiras sobre o comércio exterior brasileiro. Brasília: Instituto de Pesquisa Econômica Aplicada, 2003.

PAM. Produção Agrícola Municipal. Informação das lavouras no período de 2005 a 2012. IBGE. Disponível em: http://www.ibge.gov.br/home/estatistica/economia/pam. Acesso em: 20 de fev. de 2015.

SILVA, Sandro Pereira. Créditos do PRONAF e dinâmicas econômicas territoriais: uma análise de dados em painel para o território Vale do Mucuri - 2000 a 2007. In: XIV Seminário de Economia Mineira, 2010, Diamantina. Anais do XIV Seminário de Economia Mineira, 2010.

SOUZA FILHO, H. M.; BATALHA, M. O.. Gestão Integrada para a Agricultura Familiar - GIAF. 1. ed. São Carlo SP: EDUFSCAR, 2005. v. 1. 359p.

SOUSA, J.M.P. e VALENTE, A.S.J. Análise das Liberações dos Recursos do PRONAF Descentralização das Aplicações do Crédito Rural? Anais do XLIV Congresso SOBER, Julho de 2006.

STOCK, J. H. AND WATSON, M. W.. Combination Forecasts of Output Growth in a Seven-Country Data Set. Journal of Forecasting. Edition 23, 405-430, 2004.

WOOLDRIDGE, Jeffrey M. Introdução à econometria: uma abordagem moderna. São Paulo: Thomson Learning, [2007]. 684 p.

Renato Alves de Oliveira. Docente da Universidade Estadual de Ponta Grossa. natoliveiralves@hotmail.com

Luciano Ribeiro Bueno. Docente da Universidade Estadual de Ponta Grossa. cocovith@hotmail.com

Como citar: DE OLIVEIRA, Renato Alves; BUENO, Luciano Ribeiro. O impacto do financiamento do PRONAF sobre indicadores agrícolas nas lavouras do Estado do Paraná: uma análise de dados em painel. Redes, Santa Cruz do Sul, v. 24, n. 1, p. 292-309, jan. 2019. ISSN 1982-6745. Disponível em: https://doi.org/10.17058/redes.v24i1.7599. 